Bond University

Research Repository

\title{
Risky alcohol use and age at onset of regular alcohol consumption in relation to frontal lobe indices, reward sensitivity, and rash impulsiveness
}

Lyvers, Michael; Duff, Helen; Hasking, Penelope

Published in:

Addiction Research and Theory

DOI:

10.3109/16066359.2010.500751

Licence:

CC BY-NC-ND

Link to output in Bond University research repository.

Recommended citation(APA):

Lyvers, M., Duff, H., \& Hasking, P. (2011). Risky alcohol use and age at onset of regular alcohol consumption in relation to frontal lobe indices, reward sensitivity, and rash impulsiveness. Addiction Research and Theory, 19(3), 251-259. https://doi.org/10.3109/16066359.2010.500751

\section{General rights}

Copyright and moral rights for the publications made accessible in the public portal are retained by the authors and/or other copyright owners and it is a condition of accessing publications that users recognise and abide by the legal requirements associated with these rights.

For more information, or if you believe that this document breaches copyright, please contact the Bond University research repository coordinator 
Risky Alcohol Use and Age at Onset of Regular Alcohol Consumption in Relation to Frontal Lobe Indices, Reward Sensitivity and Rash Impulsiveness

Michael Lyvers, Ph.D.

Helen Duff, Honours

Department of Psychology

Bond University

Gold Coast Qld 4229 Australia

Corresponding Author: Michael Lyvers

Phone: (61) (75) 5952565

Fax: (61) (75) 5952540

Email: mlyvers@staff.bond.edu.au 


\begin{abstract}
Objective: Excessive alcohol consumption has been linked to predisposing traits that may reflect frontal lobe functioning, an idea explored by the present study. Method: The study recruited 132 adults of both genders aged 18-68 who completed an online questionnaire battery. Results: Two aspects of impulsivity (reward sensitivity and rash impulsiveness), as well as the Disinhibition and Executive Dysfunction indices of the Frontal Systems Behavior Scale (FrSBe), were associated with risky drinking as defined by the Alcohol Use Disorders Identification Test (AUDIT). Reward sensitivity (SR) partially mediated the association between Disinhibition and AUDIT. Higher SR predicted younger age at onset of weekly drinking (AOD). Parental alcoholism as measured by the Children of Alcoholics Screening Test was also associated with younger AOD and riskier drinking. By contrast, sensitivity to punishment (SP) was associated with later AOD in older participants, and with lower alcohol consumption in younger participants. Conclusions: The present findings suggest that several interrelated and heritable personality and neurobehavioral traits may promote earlier as well as riskier alcohol consumption.
\end{abstract}

Key Words: Alcohol ; Alcohol Abuse ; Frontal Lobe ; Impulsivity ; Reward Dependence rash impulsiveness. Addiction Research and Theory, 19(3), 251-259. https://doi.org/10.3109/16066359.2010.500751. 
Research on the etiology of problematic alcohol use has invoked neurobiological and personality variables that preceded alcohol exposure as well as neurobehavioral deficits attributed to the duration and severity of exposure (Kreek, Nielsen, Butelman, \& LaForge, 2005; Lyvers, 2000; Simons, Gaher, Correia, Hansen, \& Christopher, 2005; Varma, Basu, Malhotra, Sharma, \& Mattoo, 1994; Verdejo-García, Rivas-Péreza, López-Torrecillasa, \& Pérez-García, 2006; Volkow \& Li, 2004). Factors said to promote alcoholism include an inherited mild dysfunction of the prefrontal cortex (Spinella, 2003), personality traits such as impulsivity and neuroticism (Hair \& Hampson, 2006; Kambouropoulos \& Staiger, 2007; Schmidt, Buckner, \& Keough, 2007; Zuckerman \& Kuhlman, 2000), an early onset age for excessive alcohol use at a time of cognitive vulnerability (Crews, He, \& Hodge, 2007; Pitkänen, Lyyra, \& Pulkkinen, 2005) and the cumulative effects of prolonged high alcohol exposure (Kubota et al., 2001; Lyvers, 2000).

The "frontal lobe" hypothesis of addictions suggests that impairment of the capacity to properly evaluate rewards and punishments and to regulate and inhibit impulsive behavior are critical factors promoting the onset and maintenance of compulsive substance use (Lyvers, 2000). The prefrontal cortex regulates limbic and parietal regions (Phan et al., 2005) in a synergistic process which is compromised by excessive alcohol usage, as demonstrated in the effects of acute and chronic intoxication (Giancola, 2000; Rose \& Duka, 2007; Sullivan \& Pfefferbaum, 2005; Sullivan, Rosenbloom, \& Pfefferbaum, 2000). The orbitofrontal area is particularly implicated as it is involved in responding to reinforcers, assessing risks and inhibiting inappropriate behavioral responses (Beer, Knight, \& D'Esposito, 2006; Berlin, Rolls, \& Kischka, 2004; Hornak et al., 2004; Rolls, 2004). Early onset heavy drinking has been implicated in possible alcohol induced changes to the brain of adolescents (Crews et al., 2007; Monti et al., 2005; Verdejo-García et al., 2006; Volkow $\& \mathrm{Li}, 2004)$. Another possibility however is that mild prefrontal cortical dysfunction may predate the onset of excessive alcohol use in young people as a genetic predisposition (Agrawal \& Lynskey, 2008; Goodwin, Schulsinger, Hermansen, Guze, \& Winokur, 1973; Lyvers, Czerczyk, Follent, \& Lodge, 2009; Yacubian et al., 2007). Consistent with a genetic interpretation, early onset age of rash impulsiveness. Addiction Research and Theory, 19(3), 251-259. https://doi.org/10.3109/16066359.2010.500751. 
regular drinking is associated with trait indices of reward sensitivity (Lyvers et al.) and is also a strong predictor of life-time alcohol misuse (Pitkänen et al., 2005). Further, early onset alcoholism is associated with trait impulsivity (Pardo, Aguilar, Molinuevo, \& Torrubia, 2007) and familial alcoholism (Dom et al., 2006; Varma et al., 1994). A predisposition to excessive alcohol use in adolescents and adults may reflect interactions between the limbic system and orbitofrontal cortex (Enoch \& Goldman, 2002) that manifest as reduced behavioral inhibition and increased impulsivity (Berlin et al., 2004; Franken, van Strien, Nijs, \& Muris, 2008) prior to alcohol exposure.

Impulsivity, risk taking and rash decision making in anticipation of reward have been linked to orbitofrontal and dopaminergic system dysfunction (Berlin et al., 2004; Elliott \& Deakin, 2005; Franken et al., 2008; Schoenbaum \& Shaham, 2008; Yacubian et al., 2007). Dopaminergic neurons promote approach behavior when activated by cues predicting reward and pause in their firing when an expected reward is not received (Schultz, Tremblay, \& Hollerman, 2000; Schulz, 2001). An overactive sensitivity to reward involves reduced ability to evaluate the implications of negative outcomes, such that negative emotions aroused by errors or loss of reward are less able to influence decision making (Beer et al., 2006; Hornak et al., 2004). Failure to recognize the loss of reward, and elevated anticipation irrespective of reward probability and magnitude, have been demonstrated in patients with orbitofrontal damage (Hornak et al., 2004). Impulsive behavior and high reward sensitivity have also been associated with problematic drinking and other forms of substance abuse (Dawe, Gullo, \& Loxton, 2004; Dawe \& Loxton, 2004; Hanson, Luciana, \& Sullwold, 2008; Lyvers et al., 2009; O'Connor \& Colder, 2005; Simons et al., 2005),

Both self-report measures (Dom et al., 2006) and objective laboratory tasks (Bjork et al., 2004) have indicated a negative relationship between impulsivity and age of onset of drinking. Dawe et al. (2004) proposed that impulsivity has two distinct dimensions: reward sensitivity (SR), or the degree to which behavior tends to be motivated by the prospect of positive reinforcement, and rash impulsiveness, or difficulties with the inhibition of behavior. They suggest that the former can be measured by the Sensitivity to Punishment and Sensitivity to Reward Questionnaire (SPSRQ; 
Torrubia, Ávilab, Moltób, \& Caseras, 2001) and the latter by the Barratt Impulsiveness Scale (BIS11; Patton, Stanford, \& Barratt, 1995), suggestions implemented in the present study. In Dawe et al.'s model, SR was proposed to play a major role in the onset of regular substance use, whereas rash impulsiveness was said to promote ongoing excessive or problematic substance use reflecting orbitofrontal dysfunction and associated disinhibition of behavior (Dawe et al., 2004; Dawe, Loxton, Gullo, \& Staiger, 2007; Horn, Dolan, Elliott, Deakin, \& Woodruff, 2003; Ketzenberger \& Forrest, 2000).

A few studies have examined alcohol consumption in relation to measures of SR based on Gray's Reinforcement Sensitivity Theory (RST), such as the BIS/BAS scales (Carver \& White, 1994) where the BAS (Behavioral Activation System) Scale is an index of SR and the BIS (Behavioral Inhibition System) Scale is an index of sensitivity to punishment (SP) (Corr, 2002). Loxton and Dawe (2001) reported that BAS but not BIS scores were related to alcohol consumption as measured by the Alcohol Use Disorders Identification Test (AUDIT; Babor, de la Fuente, Saunders \& Grant, 1992), and Pardo et al. (2007) reported a positive association between BAS scores and alcohol consumption as well as a negative association between BAS scores and age at first alcohol drink. As age at first drink is likely to reflect situational factors, the present study asked for the age at which regular (i.e., weekly) drinking was initiated, because this was expected to be more sensitive to individual differences in SR and was in line with other recent findings (Lyvers et al., 2009) as well as theory (Dawe et al., 2004). Although a direct relationship between anxiety or SP and alcohol use has not been conclusively established (Loxton \& Dawe, 2001), Feil and Hasking (2008) suggested that anxiety and stress can play an important role in the development of excessive drinking. According to Kambouropoulos and Staiger (2004) individuals with high SP drink to alleviate environmentally aroused aversive states such as anxiety. Alcohol may thus be used by some to dampen an over-reactive aversive motivational system (Fowles, 1987). On the other hand, anxiety is conjectured to serve as a protective factor against disinhibited, potentially dangerous activities and behaviors that could lead to early mortality in adolescence and early adulthood (Lee, rash impulsiveness. Addiction Research and Theory, 19(3), 251-259. https://doi.org/10.3109/16066359.2010.500751. 
Wadsworth, \& Hotopf, 2006). Thus high levels of SP might be expected to protect against alcohol problems in young adults, despite the possibility of later onset of excessive drinking to alleviate negative states such as anxiety (Kambouropoulos \& Staiger, 2004). This idea was one of several investigated in the present study.

Self-report questionnaires are commonly used to assess personality traits and are increasingly regarded as an efficient means of examining hypotheses about neurobiological functioning (Verdejo-Garcia, Lawrence \& Clark, 2008). The Frontal Systems Behavior Scale (FrSBe; Grace \& Malloy, 2001) has been shown to identify dysfunction as well as subtle gradations of functioning associated with three frontal systems (dorsolateral, orbitofrontal, anterior cingulate) in both clinical and non-clinical samples (Spinella, 2007a). A previous study by Lyvers et al. (2009) found that FrSBe Disinhibition scores (designed to assess orbitofrontal dysfunction) and SPSRQSR scores were both positively correlated with scores on the AUDIT, where higher scores indicate heavier alcohol consumption and riskier drinking. Further, SR scores were negatively related to age at onset of regular alcohol use, and high risk drinkers (as defined by AUDIT) had higher FrSBe Disinhibition and SR scores compared to low risk drinkers. These findings cannot distinguish between characteristics which developed since the onset of alcohol use and inherent characteristics which preceded alcohol use (Verdejo-García et al.). However, as the findings of Lyvers et al. were obtained in a young adult sample of university undergraduates aged 18-25 years, the authors suggested that the links between SR, Disinhibition and AUDIT most likely reflected trait factors predisposing to earlier and heavier alcohol consumption rather than neurobiological effects of chronic heavy drinking. The present study recruited participants across a much broader age range, as arguably the factors promoting heavier alcohol consumption in older adults might vary from those in younger age groups (Schmidt et al., 2007; Varma et al., 1994; Welte \& Mirand, 1995). Further, the present study included the BIS-11 as an index of rash impulsiveness in addition to the SPSRQ measure of SR as per Dawe and Loxton's (2004) notion of two separable components of impulsivity. Parental alcoholism as assessed by the Children of Alcoholics Screening Test (CASTrash impulsiveness. Addiction Research and Theory, 19(3), 251-259. https://doi.org/10.3109/16066359.2010.500751. 
6; Hodgins, Maticka-Tyndale, El-Guebaly \& West, 1993) was also examined in relation to SR, Disinhibition, BIS-11, AUDIT and age at onset of regular drinking (AOD), with the expectation that trait factors related to heavier and earlier consumption (such as Disinhibition and SR) would be associated with parental alcoholism in line with presumed genetic risk factors for alcohol problems.

\section{Method}

\section{Participants}

The 132 participants were 87 females and 45 males; ages ranged 18 to 68 years $(M=36.06$, $S D=14.65$ ), of whom $35 \%$ fell within the 18 to 25 year age group (young adults). All participants were at least occasional alcohol drinkers (note that the legal drinking age in Australia is 18). The sample included 40 students from the university's undergraduate psychology participant pool who participated for course credit, and 92 members of the local community recruited through local high school teachers (snowball method; no incentive offered). In terms of education level, $80 \%$ of the sample reported that they had completed year 12 or higher. Only $1.5 \%$ of the sample said they were regular users of illicit drugs (defined as using more than three times per month), and 20\% indicated they were current tobacco smokers, consistent with Australian Institute of Health and Welfare (AIHW, 2008) national averages. The overall sample size was adequate for multiple regression as recommended by Tabachnik and Fidell (2007).

\section{Materials}

A commercial online survey program, Survey Monkey, was used to present the questionnaires described below via the internet.

The Alcohol Use Disorders Identification Test (AUDIT; Babor et al., 1992) is composed of 10 questions, including 3 quantity/frequency questions (e.g., "How often do you have a drink containing alcohol?"), 3 dependence-related items (e.g., "How often during the last year have you failed to do what was normally expected of you because of drinking?"), and 4 alcohol-related consequences or harm (e.g., "Have you or someone else been injured because of your drinking?"). AUDIT questions are scored from 0 to 4 , with an overall score ranging from $0-40$. The suggested rash impulsiveness. Addiction Research and Theory, 19(3), 251-259. https://doi.org/10.3109/16066359.2010.500751. 
cut-offs for Hazardous and Harmful drinking are 8 and 16, respectively. Internal consistency (Cronbach's alpha) and test-retest reliability are consistently reported to be high (Bergman \& Källmén, 2002; Dybek et al., 2006; Kane, Loxton, Staiger, \& Dawe, 2004; Pal, Jena, \& Yadav, 2004; Rubin et al., 2006). Convergent validity has been supported by correlations with the Michigan Alcoholism Screening Test of up to .97 (Pal et al., 2004).

The Frontal Systems Behavior Scale (FrSBe; Grace \& Malloy, 2001) Self-Rating form is a 46-item questionnaire assessing behavioral evidence of dysfunction in three major prefrontalsubcortical systems of the brain. There are three subscales: Apathy (poor initiation, reduced drive and interest), designed to assess anterior cingulate dysfunction; Disinhibition (distractibility, problems with inhibition, socially inappropriate behavior), designed to assess orbitofrontal dysfunction; and Executive Dysfunction (difficulties with planning, sequencing, working memory, and mental flexibility), designed to assess dorsolateral prefrontal dysfunction. The standard version of the Self Rating form of the FrSBe aims to measure behavioral change by obtaining pre-and postlesion ratings. For the purposes of this study and in keeping with previous research (Lyvers et al., 2009; Spinella, 2003; Verdejo-García et al., 2006) only overall scores in present time were obtained. Items are rated on a 5 point Likert scale ( $1=$ almost never, $2=$ seldom, $3=$ sometimes, $4=$ frequently, $5=$ almost always). The first 32 items represent deficits and are rated accordingly, with the final 14 positively stated items reverse scored. The magnitude of the score on each subscale indicates the degree of impairment. Factor analyses of the FrSBe in several neurological populations have supported the construct validity of the subscales (Stout, Ready, Grace, Malloy, \& Paulsen, 2003). Evidence also supports reliability (Velligan, 2002) with internal consistency from .88 to .91 and three month test-retest reliability of .78 . Diagnostic validity has been confirmed for detecting graduated degrees of symptoms of frontal lobe functioning in various clinical samples (Chiaravalloti \& DeLuca, 2003; Velligan, 2002), in substance use and abuse populations (Spinella, 2003) and in healthy individuals (Spinella, 2007a). rash impulsiveness. Addiction Research and Theory, 19(3), 251-259. https://doi.org/10.3109/16066359.2010.500751. 
The Sensitivity to Punishment and Sensitivity to Reward Questionnaire (SPSRQ; Torrubia et al., 2001) is a 48 yes-no response item questionnaire consisting of two scales: Sensitivity to Punishment (SP; 24 items, e.g. “Are you often afraid of new or unexpected situations?”), and Sensitivity to Reward (SR; 24 items, e.g. “Do you sometimes do things for quick gains?”). Dichotomous responses of either "yes" (1) or "no" (0) yield a score for each scale which is a summation of all affirmative responses. The magnitude of the score indicates the level of SR and SP. Both scales demonstrate acceptable levels of internal consistency and three month test-retest reliability (O'Connor \& Colder, 2005; Torrubia et al., 2001). Construct validity has been demonstrated in a two factor solution (Caci, Deschaux, \& Bayle, 2007; O'Connor, Colder, \& Hawk, 2004). SR has been found to be positively related to extraversion $(r=.48)$ and Gray's BAS scale $(r$ $=.43)$, and SP related to neuroticism $(r=.70)$ and Gray's BIS scale $(r=.50)$ (Sava \& Sperneac, 2006), consistent with the theoretical underpinnings of the SPSRQ.

The Barratt Impulsiveness Scale (BIS-11; Patton et al., 1995) is a 30-item self-report questionnaire designed to measure trait impulsivity. Factor analysis in a clinical population (Patton et al., 1995) and later replicated in a non-clinical, community population (Spinella, 2007b) demonstrated the construct validity of three separate impulsivity subscales: (a) non-planning impulsivity or the inability to plan and think carefully; 11 items, e.g. "I plan tasks carefully" (reverse scored item), (b) motor impulsivity or acting on the spur of the moment; 11 items, e.g. "I do things without thinking," and (c) attentional impulsivity or the inability to focus on the task at hand; 8 items, e.g. "I don't pay attention." Items are rated on a four-point Likert scale ranging from "rarely/never" to "almost always." Individual items are summed to create an overall score, with higher scores representing greater levels of impulsiveness. Research has established that the BIS-11 has strong psychometric properties in both psychiatric (Patton et al., 1995) and non-clinical populations (Spinella, 2007a) with reliability coefficients from .79 to .83 and test-retest stability over one year of .60 (Luengo, Carrillo-de-la-Pena, \& Otero, 1991). Convergent validity was established with neuropsychological measures that have demonstrated sensitivity to orbitofrontal rash impulsiveness. Addiction Research and Theory, 19(3), 251-259. https://doi.org/10.3109/16066359.2010.500751. 
prefrontal dysfunction (the Go/No-Go Test, $r=.39$, and the Antisaccade Test, $r=.48$; Spinella, 2004 ) as well as related subscales of the FrSBe (Executive Dysfunction, $r=.70$; Disinhibition, $r$ $=.60 ;$ Spinella, 2007b).

The Children of Alcoholics Screening Test-6 (CAST-6) was developed by Hodgins et al. (1993) as a short version of the CAST, a widely used 30 item yes-no scale designed to assess perceptions of parental impairment due to alcohol to identify adult children of alcoholics. The CAST-6 consists of 6 items (e.g., "Did you ever wish that a parent would stop drinking?").

Dichotomous responses of either "yes" (1) or "no" $(0)$ produce a score which is a summation of all affirmative responses. A score of 3 or more indicates a probable child of an alcoholic parent. The CAST-6 was derived from 6 items which loaded highest across clinical and non-clinical samples after independent principle components analysis (Hodgins et al.). Internal consistency and testretest stability are high, and CAST-6 scores are highly correlated with scores on the 30 -item CAST $(r=.93 ;$ Hodgins \& Shimp, 1995).

A Demographic Questionnaire asked participants to provide personal information on age, gender, years of education, current age, the age at which they started drinking weekly (AOD), illicit drug use and smoking.

\section{Procedure}

After obtaining ethical clearance from the Bond University Human Research Ethics Committee (BUHREC), recruitment of university students was undertaken via poster advertisement and sign-up sheets which directed interested participants to a room on campus with computers for completion of the online questionnaire battery. The 40 undergraduate university students participated for course credit in introductory psychology classes. Community participants were recruited through emailed invitation to local high school teachers. Each community volunteer was invited to recruit further participants from their own personal networks. No incentive was provided for community participants. All participants were required to be at least 18 years of age. Those who volunteered were provided access to the online questionnaire site. A commercial online survey rash impulsiveness. Addiction Research and Theory, 19(3), 251-259. https://doi.org/10.3109/16066359.2010.500751. 
program, Survey Monkey, was used to conduct the study. The questionnaires were completed individually online and submitted through a temporary link. Access to the questionnaires was only made available after the explanatory statement had been read and online consent had been indicated. Completion took approximately 30 minutes. All participants were de-identified by the survey program which had been designed so that answers could not be tracked to computer ISP addresses or individuals as per ethics committee requirements. Answers were automatically coded and collated into an anonymous data pool. The data were analysed with the SPSS 16 Graduate Pack statistical package.

\section{Results}

Analyses included bivariate correlations, between-subjects multivariate analyses of covariance (MANCOVA), standard multiple regressions and pathway analysis. The variables were examined separately for missing values and fit between distributions and assumptions. Six cases with missing data in the Years of Education variable were replaced with the mean. Boxplots identified several moderate outliers. Preliminary analysis for univariate outliers using $z$ scores found no extreme outliers although several cases recorded standardised scores in excess of the $\pm 3.29(p<.001)$ recommended by Tabachnik and Fidell (2007, p.73). One of these cases was also identified in the residual scatterplot and through Casewise Diagnostics with a standardised residual approaching 3.3. Tabachnik and Fidell $(2007$, p.73) advise that a few scores in excess of the recommended standardised residual are to be expected with a large sample. With the use of a $p<.001$ criterion for Mahalanobis distance this case was also identified as a multivariate outlier. Investigation of survey responses identified all outliers as being indicative of the intended population and appropriate to the sample. The size of the data set $(n=132)$ was deemed to allow retention of all cases with minimal impact. Assumptions concerning linearity and multicollinearity were investigated and met.

Normality in the scatterplot and the normality line for standardized residuals were deemed acceptable for AUDIT score as a dependent variable but indicated some departure from normality rash impulsiveness. Addiction Research and Theory, 19(3), 251-259. https://doi.org/10.3109/16066359.2010.500751. 
for AOD as expected. Deviations from normality in the AUDIT scores (skewness $=1.5$ ) were judged to not make a substantive impact on analyses due to the sample size (Tabachnik \& Fidell, 2007, p.80). Of the present sample 57.58\% reported Low Risk drinking as defined by AUDIT ( $n=$ 76), whereas one-third were defined as Hazardous drinkers $(n=44)$ and $9 \%$ scored in the Harmful range $(n=12)$. These results were consistent with the Australian Institute of Health and Welfare (AIHW, 2008) national average for alcohol consumption. The age at which participants started drinking weekly (AOD) ranged from 13 to 48 years $(M=19.18, S D=6.13)$.

Of the 132 participants only 34 were classified as children of alcoholics by the CAST6, all of whom reported an early AOD (before the age of 25). The mean AOD for children of alcoholics $(M=17.06$ years, $S D=1.89)$ was significantly earlier than for other participants $(M=$ 19.92 years, $S D=6.89), t(126)=3.77, p<.0001$ (unequal variances assumed). Of the participants classed as children of alcoholics, $56 \%$ drank at a risky level (Hazardous or Harmful) compared to $38 \%$ in the rest of the sample. The mean AUDIT score for children of alcoholics was above the AUDIT cutoff score of $8(M=9.41, S D=6.67)$ which separates Low Risk from Hazardous drinkers, whereas the mean for other participants was below $8(M=6.91, S D=5.41)$. An independent $\mathrm{t}$-test indicated that AUDIT scores were significantly higher for children of alcoholics than for other participants, $t(130)=2.17, p=.03$.

Intercorrelations were calculated among Years of Education, Age, AOD, CAST-6, AUDIT, FrSBe Apathy, Disinhibition and Executive Dysfunction, SPSRQ SP and SR, and BIS-11. These intercorrelations are shown in Table 1. The important correlations to note in Table 1 are (1) the significant positive correlations of AUDIT with FrSBe Disinhibition and Executive Dysfunction, SPSRQ SR, BIS-11 and CAST-6, and (2) the significant negative correlations of AOD with SR and CAST-6 but a positive correlation with SP. When correlations were conducted separately for younger (18-25 years) and older (26-68 years) participants, most of the significant correlations were very similar; i.e., in both age groups, AUDIT scores were significantly positively correlated with FrSBe Disinhibition and Executive Dysfunction as well as with SPSRQ SR and BIS-11. However, 
AOD was positively correlated with SP in the older age group, $r(85)=.39, p<.0001$, but not the younger age group, $r(47)=.13, p=.38$; and CAST was positively correlated with AUDIT in the older age group, $r(85)=.36, p<.001$, but not in the younger age group, $r(47)=.04$, n.s.

A standard multiple regression was performed to explore influences of trait variables of interest on AOD. The regression was performed with AOD as the criterion and seven predictor variables: CAST, FrSBe Apathy, Disinhibition and Executive Dysfunction, SPSRQ SP and SR, and BIS-11. Table 2 displays the unstandardised regression coefficients $(B)$, standardised regression coefficients $(\beta)$, semi-partial correlations $\left(S r^{2}\right), R^{2}$ and adjusted $R^{2} . R$ for regression was significant, $F(7,124)=4.86, p<.001$. The adjusted $R^{2}$ indicated that $17 \%$ of the variability in AOD was predicted by the seven factors. Although the CAST and SR both made a significant negative contribution, SP made the strongest unique contribution to AOD (see Table 2).

The hypothesis that BIS-11, SPSRQ SR, and FrSBe Disinhibition and Executive Dysfunction would vary according to AUDIT Risk Group (Low Risk, High Risk) was investigated through a 2 (Gender) x 2 (AUDIT levels) between-groups MANCOVA performed on all three FrSBe scales, the SPSRQ scales and BIS-11. Age was entered as a covariate given its relationship to most of the other variables (see Table 1). The High Risk group included both Hazardous and Harmful drinkers as defined by AUDIT. The assumption of homogeneity of variance-covariance matrices (Box's M) was not significant, indicating no violation. As the Levene's test was significant for SP and BIS-11, a more stringent alpha level was set $(p<.01)$ (Tabachnik \& Fidell, 2007). Pillai's trace indicated that the combined dependent variables were significantly affected only by the covariate age and by AUDIT risk level, $F(6,122)=5.17, p<.0001$, observed power $=.99$. There was no effect of Gender and no interaction. When the unique effects of the AUDIT grouping variable on the dependent variables were considered, all variables except Apathy and SP were significant: Disinhibition, $F(1,127)=24.89, p<.0001$, observed power $=1.00$; Executive Dysfunction, $F(1,127)=7.36, p<.01$, observed power $=.77$; BIS-11, $F(1,127)=9.46, p<.01$, rash impulsiveness. Addiction Research and Theory, 19(3), 251-259. https://doi.org/10.3109/16066359.2010.500751. 
observed power $=.86 ; \mathrm{SR}, F(1,127)=6.45, p<.01$, observed power $=.71$. The AUDIT Risk Group means are shown in Table 3.

These relationships were further investigated using AUDIT as a continuous variable. A standard multiple regression was performed with total AUDIT scores as the criterion and six predictor variables: FrSBe Apathy, Disinhibition and Executive Dysfunction, SPSRQ SP and SR, and BIS-11. Table 4 displays the standardised regression coefficients $(B)$, standardised regression coefficients $(\beta)$, the semi-partial correlations $\left(S r^{2}\right), R^{2}$ and adjusted $R^{2} . R$ for regression was significantly different from zero, $F(6,125)=11.91, p<.001$. The adjusted $R^{2}$ indicated that $33 \%$ of the variability in AUDIT was predicted by the six factors. Disinhibition made the strongest unique contribution when the variance of all other variables was controlled (see Table 4).

Disinhibition was significantly associated with AUDIT risk in the MANCOVA and was also a significant predictor of AUDIT score in the regression. Both BIS-11 and SR had a significant association with AUDIT risk in the MANCOVA but only SR was a significant predictor of AUDIT in the regression. These relationships were further investigated via a simple path analysis to determine the extent to which the influence of Disinhibition on AUDIT was mediated by either component of impulsivity (SR or BIS-11). With a significant relationship between Disinhibition and the AUDIT confirmed, BIS-11 and SR were regressed on Disinhibition followed by the regression of all three predictors (BIS-11, SR and Disinhibition) onto AUDIT. Exploratory regression with a centered interaction term for each proved nonsignificant, ruling out moderation. Figure 1 shows the path coefficients and the error variance. Although the Sobel test statistic for SR was significant $(t=$ 2.07, $p<.05$ ), indicating convincing evidence of a strong mediation pathway, the relationship change between Disinhibition and AUDIT $(\beta=.52$ to $\beta=.39)$ indicated only partial mediation. The path between Disinhibition and AUDIT, although weaker, was still significant (see Figure 1). The path coefficient between BIS-11 and AUDIT was nonsignificant $(\beta=.04)$ and the Sobel test was nonsignificant $(t=0.45, \mathrm{~ns})$. BIS-11 thus contributed little to prediction of AUDIT and did not mediate the extent to which Disinhibition determined AUDIT scores. 


\section{Discussion}

The present findings are consistent with hypothesized links between prefrontal cortex dysfunction, reward sensitivity, rash impulsiveness and alcohol related risk. Approximately one third of the variation in AUDIT scores in the current sample was attributable to the measured personality and neurobehavioral factors.

Previous research indicated that impairment of orbitofrontal inhibitory processes is behaviorally manifested by disinhibition and impulsivity (Berlin et al., 2004; Elliott \& Deakin, 2005; Franken et al., 2008; Horn et al., 2003; Schoenbaum \& Shaham, 2008; Simons et al., 2005; Spinella, 2003). Consistent with this idea, in the present study the self-report index of orbitofrontal dysfunction - FrSBe Disinhibition score - was moderately related to both SR and BIS-11 (indices of sensitivity to reward and rash impulsiveness components of impulsivity, respectively; Dawe et al., 2004), and all three variables were moderately related to AUDIT. A similar pattern was evident for FrSBe Executive Dysfunction scores, a scale designed to reflect dysfunction of the dorsolateral prefrontal cortex (Grace \& Malloy, 2001). Of the three FrSBe scales designed to detect signs of frontal lobe dysfunction in everyday life, only the Apathy scale, which was designed to reflect anterior cingulate dysfunction, was unrelated to AUDIT (although Apathy too was significantly related to SP, SR and BIS-11). The present findings thus support the hypothesized links between mild dysfunction of prefrontal (especially orbitofrontal) cortex, sensitivity to reward, rash impulsiveness and alcohol related risk. Further, CAST-6 scores were significantly positively related to AUDIT, FrSBe Disinhibition and Executive Dysfunction, SR and BIS-11, and were negatively related to AOD (such that higher CAST-6 scores were associated with earlier age of onset of weekly alcohol use). Although these associations were not strong, they are consistent with the hypothesis that inherently poorer frontal lobe functioning, and higher sensitivity to reward and rash impulsiveness, may reflect heritable risk factors for risky or harmful alcohol consumption.

In patients with orbitofrontal damage, disinhibition is associated with impulsivity and elevated sensitivity to reward (Hornak et al., 2004). In the present study such an association was rash impulsiveness. Addiction Research and Theory, 19(3), 251-259. https://doi.org/10.3109/16066359.2010.500751. 
supported in a non-clinical sample, with Disinhibition scores related to both SR and BIS-11 scores. The primary predictor of AUDIT was Disinhibition, and the strength of the relationship of Disinhibition with AUDIT was partially reduced by the filtering effect of SR as a mediator. The association between Disinhibition and rash impulsiveness (BIS-11) was also supported in this study; however, a pathway between Disinhibition, rash impulsiveness and alcohol related risk, which had been theoretically proposed and supported in previous research (Dawe et al., 2004), was not supported in the present study. Contrary to expectation, BIS-11 contributed little to prediction of AUDIT and did not mediate the extent to which Disinhibition influenced AUDIT scores. This may have been because the current sample consisted predominantly of low to medium risk drinkers, with only 12 participants reporting Harmful drinking as defined by AUDIT. Alcoholics have been found to exhibit high levels of rash impulsiveness (Ketzenberger \& Forrest, 2000) but the present nonclinical sample was not characterised by drinking at an alcoholic level. Nevertheless both BIS-11 and Executive Dysfunction scores did significantly differentiate Harmful drinkers from the other groups in the present study. The high positive correlation between BIS-11 and Executive Dysfunction, and the fact that BIS-11 and Executive Dysfunction significantly differentiated Harmful drinkers from the other groups, is consistent with a theoretical link between impaired executive control, rash impulsiveness and problematic drinking patterns (Lyvers, 2000). Rash impulsiveness (BIS-11) distinguished Harmful from Hazardous drinkers (as defined by AUDIT) in the present study, whereas SR differentiated Low Risk drinkers from the higher risk levels. This pattern of findings is consistent with the notion that each component of impulsivity has a particular role in drinking behavior, with SR promoting change from Low Risk to Hazardous drinking, and rash impulsiveness promoting and maintaining Harmful drinking (Dawe et al., 2004).

The inverse relationship between SR and the age at which an individual started drinking regularly (AOD) suggests that over-responsiveness to reward contingencies rather than rash impulsiveness influences the drinking-related choices made at younger age levels (Crews et al., 2007; Loxton \& Dawe, 2001; Monti et al., 2005; Pardo et al., 2007; Volkow \& Li, 2004). 
Conversely the positive relationship between AOD and SP implies a possible protective influence such that trait anxiety may promote delay of regular alcohol use (Feil \& Hasking, 2008; Varma et al., 1994). Of all variables, SP made the strongest unique contribution to AOD in the overall sample. However when younger (18-25) and older (26-68) adults were examined separately, an association between SP and AOD was only found in the older adults, suggesting cohort related differences. The negative relationship between the CAST-6 and AOD, and the positive relationship of CAST- 6 with AUDIT, supports research suggesting a genetic predisposition to heavier and earlier alcohol consumption, possibly involving impulsivity and reward sensitivity as heritable traits (Agrawal \& Lynskey, 2008; Dawe et al., 2007; Dom et al., 2006; Goodwin et al., 1973; Kambouropoulos \& Staiger, 2004; Varma et al., 1994). Thus CAST-6 was significantly, albeit weakly, related to SR and BIS-11 as well as Disinhibition and Executive Dysfunction.

A limitation of the present study was that although the sample included a broad age range, it was predominantly female and relatively well-educated. In order to confirm and extend the present findings, further research is required in a larger and broader sample that exhibits parameters more consistent with the general population. Ideally, longitudinal studies should be conducted in adolescents to establish whether the traits associated with alcohol use, as reported above, truly reflect an inherent predisposition to earlier and heavier alcohol consumption rather than consequences thereof.

In conclusion, the present findings are consistent with the hypothesis that a group of heritable, interrelated personality and neurobehavioral traits promote earlier and riskier alcohol consumption, and that such traits may to some extent reflect frontal lobe (particularly orbitofrontal) functioning. The popular notion of an "addictive personality" may have little foundation, but the role of premorbid neurobehavioral traits in substance abuse risk is a topic that demands extensive research attention. rash impulsiveness. Addiction Research and Theory, 19(3), 251-259. https://doi.org/10.3109/16066359.2010.500751. 


\section{References}

Agrawal, A., \& Lynskey, M. T. (2008). Are there genetic influences on addiction: Evidence from family, adoption and twin studies. Addiction, 103, 1069-1081.

Australian Institute of Health \& Welfare (2008). 2007 National Drug Strategy Household Survey: First results (Cat. no. PHE 98. Drug Statistics Series number 20). Canberra: AIHW.

Babor, T. F., de la Fuente, J. R., Saunders, J. B., \& Grant, M. (1992). Alcohol Use Disorders Identification Test. Geneva: World Health Organisation.

Beatty, W.W., Katzung, V.M., Moreland, V.J., \& Nixon, S.J. (1995). Neuropsychological performance of recently abstinent alcoholics and cocaine abusers. Drug \& Alcohol Dependence, 37, 247-253.

Beer, J. S., Knight, R. T., \& D'Esposito, M. (2006). Integrating emotion and cognition: The role of the frontal lobes in distinguishing between helpful and hurtful emotion. Psychological Science, 17, 448-453.

Bergman, H., \& Källmén, H. (2002). Alcohol use among Swedes and a psychometric evaluation of the Alcohol Use Disorders Identification Test. Alcohol and Alcoholism, $37,245-251$.

Berlin, H. A., Rolls, E. T., \& Kischka, U. (2004). Impulsivity, time perception, emotion and reinforcement sensitivity in patients with orbitofrontal cortex lesions. Brain, 127, 1108-1126.

Bjork, J. M., Hommer, D. W., Grant, S. J., \& Danube, C. (2004). Impulsivity in abstinent alcohol-dependent patients: Relation to control subjects and type 1-/type 2-like traits. Alcohol, 34, 133-150.

Caci, H., Deschaux, O., \& Bayle, F. J. (2007). Psychometric properties of the French versions of the BIS/BAS scales and the SPSRQ. Personality and Individual Differences, 42, 987-998. rash impulsiveness. Addiction Research and Theory, 19(3), 251-259. https://doi.org/10.3109/16066359.2010.500751. 
Carver, C. S., \& White, T. L. (1994). Behavioral inhibition, behavioral activation, and affective responses to impending reward and punishment: The BIS/BAS Scales. Journal of Personality and Social Psychology, 67, 319-333.

Chiaravalloti, N. D., \& DeLuca, J. (2003). Assessing the behavioral consequences of multiple sclerosis: An application of the Frontal Systems Behavior Scale (FrSBe). Cognitive and Behavioral Neurology, 16, 54-67.

Corr, P. J. (2002). J.A. Gray's Reinforcement Sensitivity Theory: Tests of the joint subsystems hypothesis of anxiety and impulsivity. Personality and Individual Differences, 33, 511-532.

Crews, F., He, J., \& Hodge, C. (2007). Adolescent cortical development: A critical period of vulnerability for addiction. Pharmacology Biochemistry and Behavior, 86, 189-199.

Dawe, S., Gullo, M., J., \& Loxton, N. J. (2004). Reward drive and rash impulsiveness as dimensions of impulsivity: Implications for substance misuse. Addictive Behaviors, 29, 1389-1405.

Dawe, S., \& Loxton, N. J. (2004). The role of impulsivity in the development of substance use and eating disorders. Neuroscience \& Biobehavioral Reviews, 28, 343-351.

Dawe, S., Loxton, N. J., Gullo, M. J., Staiger, P. K., Kambouropoulos, N., Perdon, L., et al. (2007). The role of impulsive personality traits in the initiation, development and treatment of substance misuse problems. In P. M. Miller \& D. J. Kavanagh (Eds.), Translation of addictions science into practice (1st ed., pp.321-340). Amsterdam: Pergamon.

Dom, G., Hulstijin, W., \& Sabbe, B. (2006). Differences in impulsivity and sensation seeking between early-and late-onset alcoholics. Addictive Behaviors, 31, 298-308.

Dybek, I., Bischof, G., Grothues, J., Reinhardt, S., Meyer, C., Hapke, U., et al. (2006). The reliability and validity of the Alcohol Use Disorders Identification Test (AUDIT) in a German general practice population sample. Jounal of Studies on Alcohol, 67, 473-481.

Elliott, R., \& Deakin, B. (2005). Role of the orbitofrontal cortex in reinforcement processing rash impulsiveness. Addiction Research and Theory, 19(3), 251-259. https://doi.org/10.3109/16066359.2010.500751. 
and inhibitory control: Evidence from functional magnetic resonance imaging studies in healthy human subjects. International Review of Neurobiology, 65, 89-116.

Enoch, M.-A., \& Goldman, D. (2002). Molecular and cellular genetics of alcohol addiction. In K. Davis, L., D. Charney, J. T. Coyle \& C. Nemeroff (Eds.), Neuropsychopharmacology: The Fifth Generation of Progress (5th ed., pp. 1413-1423). Philadelphia: Williams \& Wilkins.

Feil, J., \& Hasking, P. (2008). The relationship between personality, coping strategies and alcohol use. Addiction Research and Theory, 16, 526-537.

Fowles, D. C. (1987). Application of a behavioral theory of motivation to the concepts of anxiety and impulsivity. Journal of Research in Personality, 21, 417-435.

Franken, I. H. A., \& Muris, P. (2006). Gray's impulsivity dimension: A distinction between reward sensitivity versus rash impulsiveness. Personality and Individual Differences, 40, 1337-1347.

Franken, I. H. A., van Strien, J. W., Nijs, I., \& Muris, P. (2008). Impulsivity is associated with behavioral decision-making deficits. Psychiatry Research, 158, 155-163.

Giancola, P. R. (2000). Executive functioning: A conceptual framework for alcohol-related aggression. Experimental \& Clinical Psychopharmacology, 8, 576-597.

Goodwin, D. W., Schulsinger, F., Hermansen, L., Guze, S. B., \& Winokur, G. (1973). Alcohol problems in adoptees raised apart from alcoholic biological parents. Archives of General Psychiatry, 28, 238-243.

Grace, J., \& Malloy, P. F. (2001). Frontal Systems Behavior Scale. Professional manual. Lutz, FL: Psychological Assessment Resources, Inc.

Hair, P., \& Hampson, S. E. (2006). The role of impulsivity in predicting maladaptive behavior among female students. Personality and Individual Differences, 40, 943-952.

Hanson, K., Luciana, M., \& Sullwold, K. (2008). Reward-related decision-making deficits and elevated impulsivity among MDMA and other drug users. Drug and Alcohol Dependence, 96, 99-110. rash impulsiveness. Addiction Research and Theory, 19(3), 251-259. https://doi.org/10.3109/16066359.2010.500751. 
Heaton, R. K., Chelune, G. L., Talley, J. L., Kay, G. G., \& Curtis, G. (1993). Wisconsin Card Sort Test (WCST) manual revised and expanded. Odessa, FL: Psychological Assessment Resources.

Hodgins, D. C., Maticka-Tyndale, E., El-Guebaly, N., \& West, M. (1993). The CAST-6: Development of a short-form of the Children of Alcoholics Screening Test. Addictive Behaviors, 18, 337-345.

Hodgins, D. C., \& Shimp, L. (1995). Identifying adult children of alcoholics: Methodological review and a comparison of the CAST-6 with other methods. Addiction, 90, 255-267.

Horn, N. R., Dolan, M., Elliott, R., Deakin, J. F. W., \& Woodruff, P. W. R. (2003). Response inhibition and impulsivity: An fMRI study. Neuropsychologia, 41, 1959-1966.

Hornak, J., Bramham, J., Rolls, E. T., Morris, R. G., Bullock, P. R., Polkey, C. E., et al. (2004). Reward-related reversal learning after surgical excisions in orbito-frontal or dorsolateral prefrontal cortex in humans. Journal of Cognitive Neuroscience, 16, 463-478.

Kambouropoulos, N., \& Staiger, P. K. (2004). Reactivity to alcohol-related cues:

Relationship among cue type, motivational processes, and personality. Psychology of Addicitve Behaviors, 18, 275-283.

Kambouropoulos, N., \& Staiger, P. K. (2007). Personality, behavioral and affective characteristics of hazardous drinkers. Personality and Individual Differences, 42, 213-224.

Kane, T. A., Loxton, N. J., Staiger, P. K., \& Dawe, S. (2004). Does the tendency to act impulsively underlie binge eating and alcohol use problems? An empirical investigation. Personality and Individual Differences, 36, 83-94.

Ketzenberger, K. E., \& Forrest, L. (2000). Impulsiveness and compulsiveness in alcoholics and non-alcoholics. Addictive Behaviors, 25, 791-795.

Kreek, M. J., Nielsen, D. A., Butelman, E. R., \& LaForge, S. K. (2005). Genetic influences on impulsivity, risk taking, stress responsivity and vulnerability to drug abuse and addiction. Nature Neuroscience, 8, 1450-1457. rash impulsiveness. Addiction Research and Theory, 19(3), 251-259. https://doi.org/10.3109/16066359.2010.500751. 
Kubota, M., Nakazaki, S., Hirai, S., Saeki, N., Yamaura, A., \& Kusaka, T. (2001). Alcohol consumption and frontal lobe shrinkage: Study of 1432 non-alcoholic subjects. Journal of Neurology, Neurosurgery and Psychiatry, 71, 104-106.

Lee, W. E., Wadsworth, M. E., \& Hotopf, M. (2006). The protective role of trait anxiety: A longitudinal cohort study. Psychological Medicine, 36, 345-351.

Loxton, N. J., \& Dawe, S. (2001). Alcohol abuse and dysfunctional eating in adolescent girls: The influence of individual differences in sensitivity to reward and punishment. International Journal of Eating Disorders, 29, 455-462.

Luengo, M. A., Carrillo-de-la-Pena, M. T., \& Otero, J. M. (1991). The components of impulsiveness: A comparison of the I.7 Impulsiveness Questionnaire and the Barratt Impulsiveness Scale. Personality and Individual Differences,, 12, 657-667.

Lyvers, M. (2000). "Loss of control" in alcoholism and drug addiction: A neuroscientific interpretation. Experimental and Clinical Psychopharmacology, 8, 225-249.

Lyvers, M., Czerczyk, C., Follent, A., \& Lodge, P. (2009). Disinhibition and reward sensitivity in relation to alcohol consumption by university undergraduates. Addiction Research \& Theory, in press.

Monti, P. M., Miranda, R., Nixon, K., Sher, K. J., Swartzwelder, H. S., Tapert, S. F., et al. (2005). Adolescence: Booze, brains, and behavior. Alcoholism: Clinical and Experimental Research, 29, 207-220.

O'Connor, R. M., \& Colder, C. R. (2005). Predicting patterns in first-year college students through motivational systems and reasons for drinking. Psychology of Addictive Behaviors, 19, 10-20.

O'Connor, R. M., Colder, C. R., \& Hawk, L. W. (2004). Confirmatory factor analysis of the Sensitivity to Punishment and Sensitivity to Reward Questionnaire. Personality and Individual Differences, 37, 985-1002.

Oltmanns, T., F., \& Emery, R., E. (2007). Abnormal psychology (Fifth ed.). New Jersey: rash impulsiveness. Addiction Research and Theory, 19(3), 251-259. https://doi.org/10.3109/16066359.2010.500751. 
Pearson, Prentice Hall.

Pal, H. R., Jena, R., \& Yadav, D. (2004). Validation of the Alcohol Use Disorders Identification Test (AUDIT) in urban community outreach and de-addiction center samples in North India. Journal of Studies on Alcohol, 65, 794-797.

Pardo, Y., Aguilar, R., Molinuevo, B., \& Torrubia, R. (2007). Alcohol use as a behavioral sign of disinhibition: Evidence from J.A. Gray's model of personality. Addictive Behaviors, 32, 2398-2403.

Patton, J. H., Stanford, M. S., \& Barratt, E. S. (1995). Factor structure of the Barratt Impulsiveness Scale. Journal of Clinical Psychology, 51, 768 -774.

Phan, K. L., Fitzgerald, D. A., Nathan, P. J., Moore, G. J., Uhde, T. W., \& Tancer, M. E. (2005). Neural substrates for voluntary suppression of negative affect: A functional magnetic resonance imaging study. Biological Psychiatry, 57, 210-219.

Pitkänen, T., Lyyra, A.-L., \& Pulkkinen, L. (2005). Age of onset of drinking and the use of alcohol in adulthood: A follow-up study from age 8-42 for females and males. Addiction, 100, 652-661.

Rolls, E. T. (2004). The functions of the orbitofrontal cortex. Brain and Cognition, 55, 11-29.

Rose, A. K., \& Duka, T. (2007). The influence of alcohol on basic motoric and cognitive disinhibition. Alcohol and Alcoholism, 42, 544-551.

Rubin, A., Migneault, J. P., Marks, L., Goldstein, E., Ludena, K., \& Friedman, R. H. (2006). Automated telephone screening for problem drinking. Journal of Studies on Alcohol, $67,454-458$.

Sava, F. A., \& Sperneac, A.-M. (2006). Sensitivity to reward and sensitivity to punishment rating scales: A validation study on the Romanian population. Personality and Individual Differences, 41, 1445-1456.

Schmidt, N. B., Buckner, J. D., \& Keough, M. E. (2007). Anxiety sensitivity as a prospective predictor of alcohol use disorders. Behavior Modification, 31, 202-219. rash impulsiveness. Addiction Research and Theory, 19(3), 251-259. https://doi.org/10.3109/16066359.2010.500751. 
Schoenbaum, G., \& Shaham, Y. (2008). The role of the orbitofrontal cortex in drug addiction: A review of preclinical studies. Biological Psychiatry, 63, 256-262.

Schultz, W., Tremblay, L., \& Hollerman, J. R. (2000). Reward processing in primate orbitofrontal cortex and basal ganglia. Cerebral Cortex, 10, 272-284.

Schulz, W. (2001). Reward signalling by dopamine neurons. Neuroscientist, 7, 293-302.

Simons, J. S., Gaher, R. M., Correia, C. J., Hansen, C. L., \& Christopher, M. S. (2005). An affective-motivational model of marijuana and alcohol problems among college students. Psychology of Addicitve Behaviors, 19, 326-334.

Spinella, M. (2003). Relationship between drug use and prefrontal-associated traits. Addiction Biology, 8, 67-74.

Spinella, M. (2004). Neurobehavioral correlates of impulsivity: Evidence of prefrontal involvement. International Journal of Neuroscience, 114, 95-104.

Spinella, M. (2007a). Measuring the executive regulation of emotion with self-rating scales in a nonclinical population. Journal of General Psychology, 134, 101-111.

Spinella, M. (2007b). Normative data and a short form of the Barratt Impulsiveness Scale. International Journal of Neuroscience, 117, 359-368.

Stout, J. C., Ready, R. E., Grace, J., Malloy, P. F., \& Paulsen, J. S. (2003). Factor analysis of the Frontal Systems Behavior Scale (FrSBe). Assessment, 10, 79-85.

Stuss, D. T., \& Anderson, V. (2004). The frontal lobes and theory of mind: Developmental concepts from adult focal lesion research. Brain and Cognition, 55, 69-83.

Sullivan, E. V., \& Pfefferbaum, A. (2005). Neurocircuitry in alcoholism: A substrate of disruption and repair. Psychopharmacology, 180, 583-594.

Sullivan, E. V., Rosenbloom, M., \& Pfefferbaum, A. (2000). Pattern of motor and cognitive deficits in detoxified alcoholic men. Alcoholism: Clinical and Experimental Research, 24, 611-621.

Tabachnik, B. G., \& Fidell, L. S. (2007). Using multivariate statistics (5th ed.). Boston MA: rash impulsiveness. Addiction Research and Theory, 19(3), 251-259. https://doi.org/10.3109/16066359.2010.500751. 
Pearson Education.

Torrubia, R., Ávila, C., Moltó, J., \& Caseras, X. (2001). The Sensitivity to Punishment and Sensitivity to Reward Questionnaire (SPSRQ) as a measure of Gray's anxiety and impulsivity dimensions. Personality and Individual Differences, 31, 837-862.

Varma, V. K., Basu, D., Malhotra, A., Sharma, A., \& Mattoo, S. K. (1994). Correlates of early-and late-onset alcohol dependence. Addictive Behaviors, 19, 609-619.

Velligan, D. (2002). Frontal Systems Behavior Scale in schizophrenia: Relationships with psychiatric symptomatology, cognition and adaptive function. Psychiatry Research, $113,227-236$.

Verdejo-García, A., Lawrence, A. J., \& Clark, L. (2008). Impulsivity as a vulnerability marker for substance-use disorders: Review of findings from high-risk research, problem gamblers and genetic association studies. Neuroscience \& Biobehavioral Reviews, 32, 777-810.

Verdejo-García, A., Rivas-Péreza, C., López-Torrecillasa, F., \& Pérez-García, M. (2006). Differential impact of severity of drug use on frontal behavioral symptoms. Addictive Behaviors, 31, 1373-1382.

Volkow, N. D., \& Li, T. K. (2004). Drug addiction: The neurobiology of behavior gone awry. Nature Reviews Neuroscience, 5, 963-970.

Welte, J. W., \& Mirand, A. L. (1995). Drink, problem drinking and life stressors in the elderly general population. Journal of Studies on Alcohol, 56, 67-73.

Yacubian, J., Sommer, T., Schroeder, K., Gläscher, J., Kalisch, R., Leuenberger, B., et al. (2007). Gene-gene interaction associated with neural reward sensitivity. Proceedings of the National Academy of Sciences, 104, 8125-8130.

Zuckerman, M., \& Kuhlman, M. (2000). Personality and risk-taking: Common biosocial factors. Journal of Personality, 68, 999-1029. rash impulsiveness. Addiction Research and Theory, 19(3), 251-259. https://doi.org/10.3109/16066359.2010.500751. 


\section{Table 1}

Intercorrelations among Years of Education (Educ), Age, Age of Onset of weekly Drinking (AOD), CAST-6 (CAST), AUDIT (AUD), FrSBe-Apathy (Apath), FrSBE-Disinhibition (Disin), FrSBeExecutive Dysfunction (Exec), SP and SR from the SPSRQ, and BIS-11.

\section{Educ Age AOD CAST AUD Apath Disin Exec SP SR}

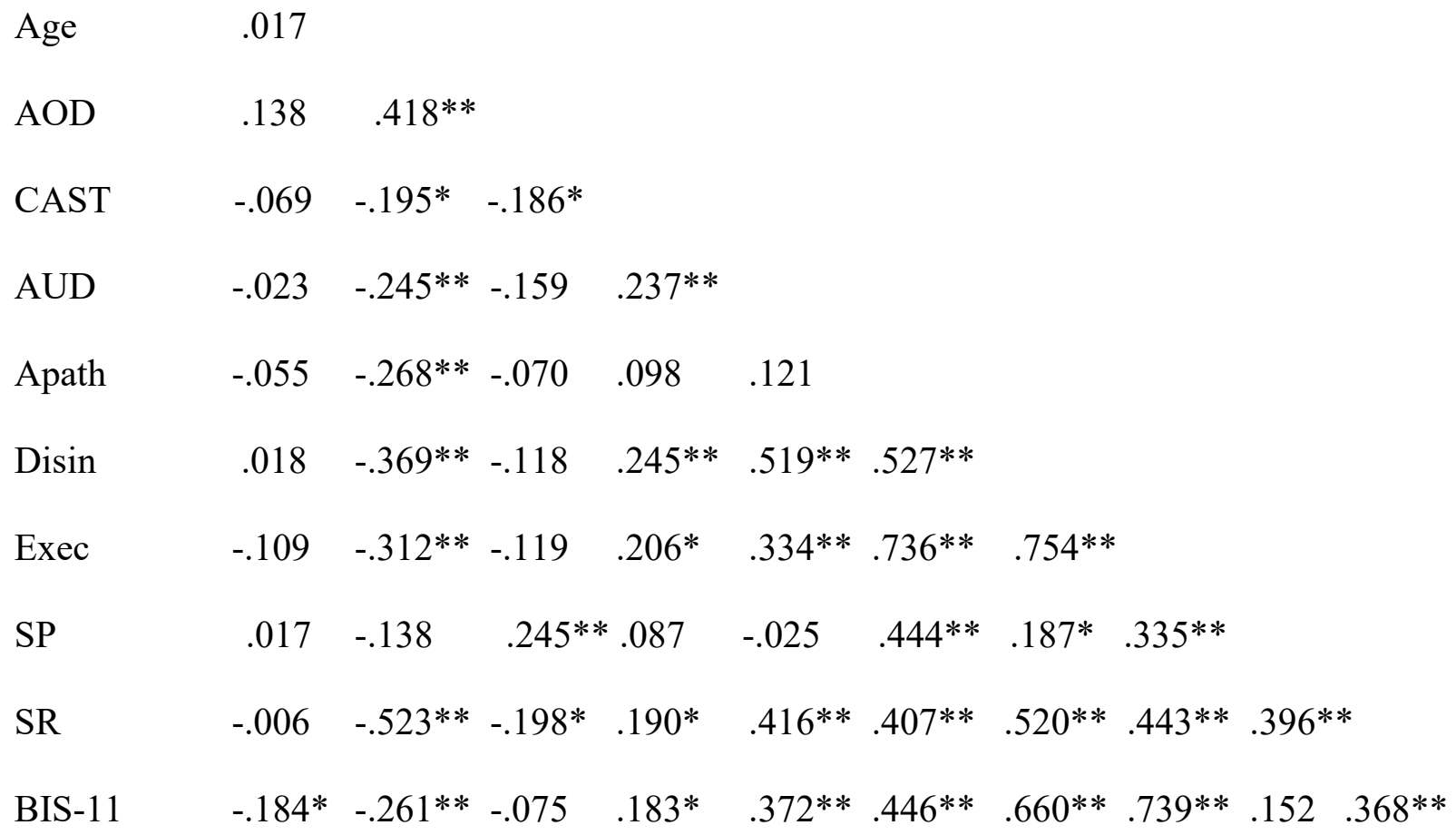

$* * \mathrm{p}<.01 * \mathrm{p}<.05$ 


\section{Table 2}

Standard multiple regression of the CAST-6, FrSBe Apathy, Disinhibition and Executive Dysfunction, SPSRQ Sensitivity to Punishment and Sensitivity to Reward, and BIS-11 Impulsiveness on Age of Onset of weekly Drinking.

\begin{tabular}{lcccc}
\hline \multicolumn{1}{c}{ Variables } & B & Std. Err & $\beta$ & $\mathrm{Sr}^{2}$ \\
\hline CAST-6 & -.495 & .248 & $-.165^{*}$ & -.03 \\
Apathy & -.090 & .129 & -.088 & -.003 \\
Disinhibition & .130 & .117 & .148 & .007 \\
Executive Dysfunction & -.165 & .132 & -.222 & -.01 \\
Sensitivity to Punishment & .477 & .098 & $.459 * * *$ & .15 \\
Sensitivity to Reward & -.479 & .144 & $-.334 * *$ & -.08 \\
Impulsiveness & .068 & .075 & .113 & .005 \\
& & & & Adjusted $R^{2}=.171$ \\
& & & & $R^{2}=.46^{* * *}$ \\
& $* 0 * 05$ & &
\end{tabular}




\section{Table 3}

Means (SD) for FrSBe Disinhibition (Disin), FrSBe Executive Dysfunction (Exec), SPSRQ

Sensitivity to Reward (SR), and Impulsiveness (BIS-11) as a function of AUDIT Risk Group.

AUDIT Risk Group: $\quad$ Low Risk $(n=76) \quad$ High Risk $(n=56)$

$\begin{array}{lcc}\text { Disin } & 26.72(5.74) & 33.34(6.79) \\ \text { Exec } & 33.68(7.79) & 38.32(8.17) \\ \text { SR } & 9.45(4.23) & 12.48(3.72) \\ \text { BIS-11 } & 58.07(8.62) & 64.05(11.32)\end{array}$




\section{Table 4}

Standard multiple regression of FrSBe Apathy, Disinhibition and Executive Dysfunction, SPSRQ

Sensitivity to Punishment and Sensitivity to Reward, and BIS-11 Impulsiveness on the AUDIT.

\begin{tabular}{|c|c|c|c|c|}
\hline Variables & B & Std. Err & $\beta$ & $\mathrm{Sr}^{2}$ \\
\hline Apathy & -.212 & .110 & -.281 & -.002 \\
\hline Disinhibition & .365 & .100 & $.437 * * *$ & .07 \\
\hline Executive Dysfunction & .021 & .113 & .030 & .0001 \\
\hline Sensitivity to Punishment & -.146 & .084 & -.148 & -.06 \\
\hline Sensitivity to Reward & .403 & .122 & $.295 * *$ & .06 \\
\hline Impulsiveness & .041 & .064 & .072 & .002 \\
\hline & & & & $\begin{array}{r}R^{2}=.364 \\
\text { Adjusted } R^{2}=.333 \\
R=.603^{* * *}\end{array}$ \\
\hline
\end{tabular}




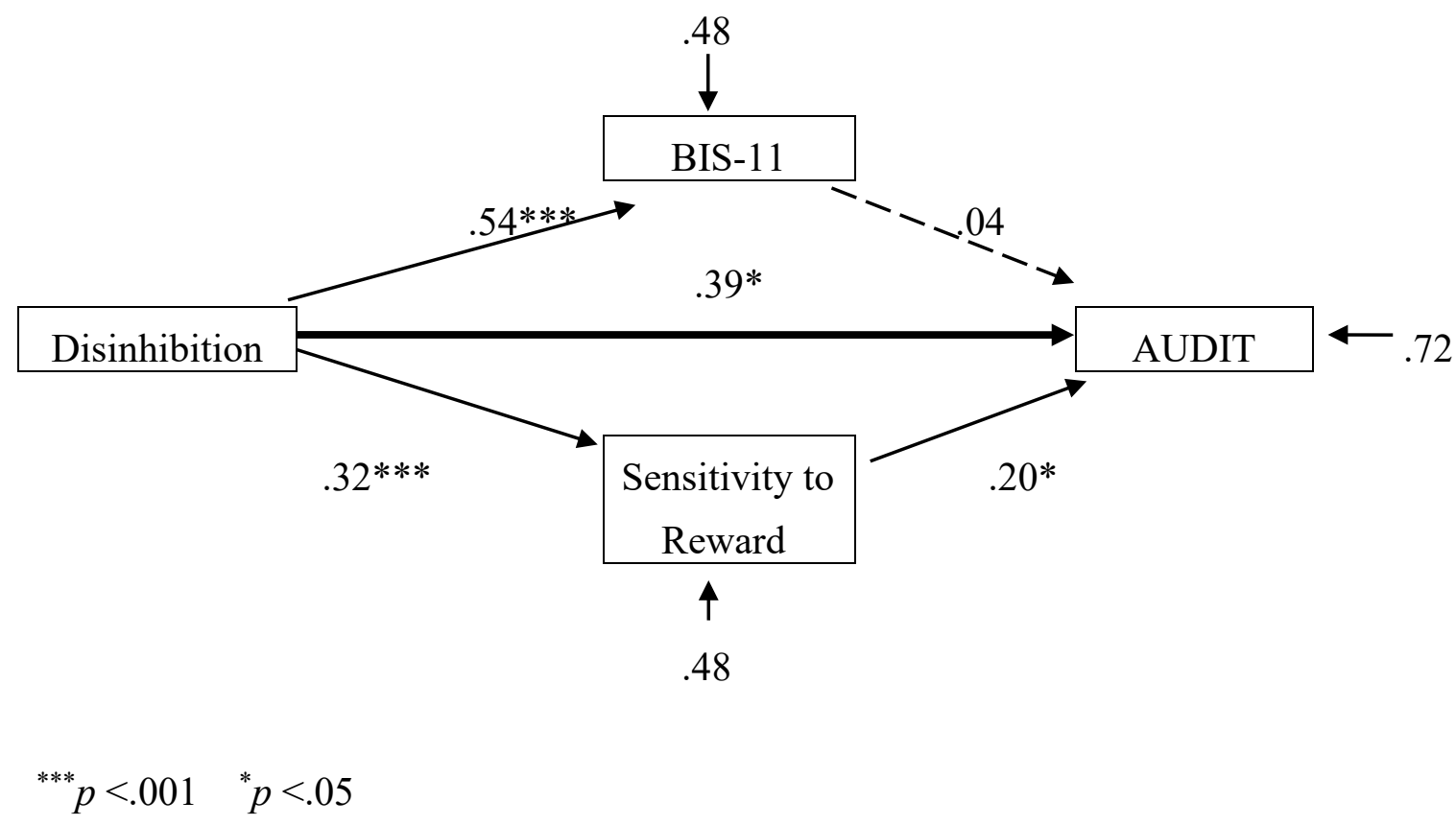

Figure 1. Path coefficients for the associations between FrSBe Disinhibition and AUDIT, with confirmed partial mediation by SPSRQ Sensitivity to Reward and unconfirmed mediation by BIS-11. Standardised betas are shown for all paths. rash impulsiveness. Addiction Research and Theory, 19(3), 251-259. https://doi.org/10.3109/16066359.2010.500751. 